\title{
LA CONVIVENCIA X VIOLENCIA EN LA ESCUELA: UN DESAFÍO
} DE LA CONTEMPORANEIDAD

\section{PRESENTACIÓN}

En esta sección, la temática "convivencia X violencia" se aborda desde determinadas contingencias que, aunque no restrictas al universo de la escuela, encontrase ahí un importante y valioso hogar de manifestación. Importante porque en la escuela, las emociones que se ven de relaciones entre personas presentan a los estudiantes con intensidad sui generis, lo que se muestra en la construcción de su moralidad.

Valioso, pues, esta condición da oportunidades a la escuela, en la medida que se elevan la convivencia al status de objeto sistemático para reflexión, el desenvolvimiento de actitudes intencionales y conocidas de eficacia, que visan la formación de valores morales que deseamos.

Para esto, no es posible prescindir, como nos demuestra la literatura mundial, de una formación de profesores capaces de subvenciónalos, para que puedan trabajar con la cuestión de la convivencia como un valor, libertando lo alumnado, así, de las amarillas que los prenden las manos frente a una tarea de superar los problemas de convivencia, que siguen en una insuficiencia de valores morales. Tornase facto la tarea de comprender la dinámica de desenvolvimiento moral y de los procesos de resolución de conflictos entre niños y adolescentes para que podremos intervenir.

De la misma forma, traemos la reflexión sobre formas no esperadas de violencia que pasan fuera de las paredes de la escuela, como el ciberbullying y otras formas de agresión y, otra vez, las formas por las cuales la sociedad moderna puede enfrentar lo desafío de la formación humana - justa, generosa, respetosa - en el mundo real o virtual.

Estos son uno de los puntos cruciales destacados en esta sección. 
Articulo 01: Bullying e ciberbullying: cuando los valores morales nos faltan y la convivencia se abala.

Articulo 02: Cuando la violencia virtual nos atinge: los programas de educación para la superación del ciberbullying y otras agresiones virtuales.

Articulo 03: Los conflictos entre alumnos de 8 y 9 años - la provocación y reacción al comportamiento perturbador: Lo que educadores deben saber.

Luciene Regina Paulino TOGNETTA ${ }^{1}$

Darlene Ferraz KNOENER ${ }^{2}$

Sanderli Ap. Bicudo BOMFIM ${ }^{3}$

Sandra Trambaiolli DE NADAI ${ }^{4}$

Thais Cristina Leite BOZZA ${ }^{5}$

Telma Pileggi VINHA ${ }^{6}$

Lívia Maria Ferreira da SILVA ${ }^{7}$

${ }^{1}$ Universidade Estadual Paulista (Unesp), Araraquara - SP - Brasil. Professora do Departamento de Psicologia da Educação Pedagoga. Líder do GEPEM - UNESP/UNICAMP. E-mail: lrpaulino@uol.com.br

${ }^{2}$ Universidade Estadual Paulista (Unesp), Araraquara - SP - Brasil. Mestranda em Educação Escolar. Membro do GEPEM - UNESP/UNICAMP. E-mail: darlene.knoener@gmail.com

${ }^{3}$ Universidade Estadual Paulista (Unesp), Araraquara - SP - Brasil. Mestranda em Educação Escolar. Membro do GEPEM - UNESP/UNICAMP. E-mail: sanderli.bicudo@gmail.com

${ }^{4}$ Universidade Estadual Paulista (Unesp), Araraquara - SP - Brasil. Mestranda em Educação Escolar. Membro do GEPEM - UNESP/UNICAMP.. E-mail: sctnadai@ hotmail.com

${ }^{5}$ Universidade Estadual de Campinas (Unicamp), Campinas - SP - Brasil. Doutoranda em Educação na área de Psicologia. Membro do GEPEM - UNESP/UNICAMP. E-mail: thaisbozza@ hotmail.com

${ }^{6}$ Universidade Estadual de Campinas (Unicamp), Campinas - SP - Brasil. Professora do departamento de psicologia educacional da UNICAMP. Membro do Laboratório de Psicologia Genética da UNICAMP e líder do GEPEM - UNESP/UNICAMP. E-mail: telmavinha@uol.com.br

${ }^{7}$ Doutora em Educação pela Unicamp, com estágio sanduíche na Harvard Graduate School of Education. Membro do Laboratório de Psicologia Genética da UNICAMP e do GEPEM - UNESP/UNICAMP. Email: liviamfsilva@gmail.com 